\title{
CASES DEMONSTRATED AT THE M.R.C.P. CLASS.
}

\section{Infective Arthritis.}

Case II.-The second patient was a girl aged 17 , also with an arthritis. She had had pain, swelling and limitation of movement in the right shoulder for three years, followed nine months ago by a similar condition in both elbows and in one metacarpophalangeal joint in the left hand. The initial condition had followed "rheumatic fever" in which the patient had had a large number of joints swollen and tender, and all had got well except the right shoulder. The affected joints were scarcely tender or swollen, but the muscles around showed much wasting; and in the elbows in particular movement was free up to a point in flexion and extension when locking suddenly occurred. The point at issue was again one of diagnosis. Adopting the classification of the causes of chronic arthritis used in the previous case this one appeared to be one of rheumatoid or of infective arthritis. Tubercle was suggested, but tubercle never involves so many of the large joints as were affected in this case, and the patient appeared so very fit. Against the diagnosis of rheumatoid arthritis were many points. The patient showed no constitutional disturbances such as are met in rheumatoid arthritis, and, e.g., had lost no weight ; only one of the smaller joints of the fingers was involved; and the more affected joints showed none of the typical periarticular changes of rheumatoid arthritis. At the same time there was evidently bony involvement to have caused the locking of the elbows. (X-rays showed a hypertrophic osteitis as the cause of this.) So evidently we were dealing with a case of infective arthritis following a history of acute infection. The other point for discussion was the causative organism. The various foci were discussed and a gonococcal cause was suggested, but none of the more classical joints involved by this organism were involved, e.g., the temporomandibular or sternoclavicular joints. Investigations had excluded this entirely, and it was thought that a streptococcal infection was probably the cause.

\section{Purpura.}

Case III.- The third case was a man aged 32, who had been in perfect health till two days previously when he had developed a profuse purpuric rash below both knees. He still had smaller and larger hæmorrhagic areas to be seen, and some fresh patches had appeared within the last twenty-four hours. The case was brought in order to discuss the causes of purpura. The causes suggested were either the old-fashioned ones, viz., purpura simplex, purpura hæmorrhagica, purpura rheumatica and Henoch's purpura; or else a grouping into thrombocytopenic and non-thrombocytopenic purpura. The first classification was criticized as it only gives an idea of the type and severity of the purpura but no idea of the cause. The only value of this classification is to draw attention to Henoch's purpura and to differentiate this from intussusception. A more rational classification based on the cause was discussed, viz.: (a) Infective, such as the malignant forms of acute specific fevers, malaria, yellow fever, septicæmias, malignant endocarditis; (b) Toxic (i) bacterial in origin as from an acute streptococcal focus or food poisoning; (ii) non-bacterial as in diabetes, jaundice, gout, nephritis; (c) drugs such as salicylates, antipyrin or sera; $(d)$ blood diseases such as leukemia, aplastic anæmia, pernicious anæmia, or primary thrombocytopenia, and $(e)$ cachectic, malignant disease, marasmus, $\$ c$. 